\title{
Plasmid selection in Escherichia coli using an endogenous essential gene marker Shan Goh ${ }^{1}$ and Liam Good*1,2
}

\author{
Address: ${ }^{1}$ Department of Cell and Molecular Biology, Karolinska Institute, Stockholm, SE-17177, Sweden and ${ }^{2}$ Department of Pathology and \\ Infectious Diseases, Royal Veterinary College, University of London, AL9 7TF, UK \\ Email: Shan Goh - shan.goh@ki.se; Liam Good* - lgood@rvc.ac.uk \\ * Corresponding author
}

Published: II August 2008

BMC Biotechnology 2008, 8:61 doi:|0.1 |86/|472-6750-8-61
Received: 4 April 2008

Accepted: II August 2008

This article is available from: http://www.biomedcentral.com//472-6750/8/6 I

(C) 2008 Goh and Good; licensee BioMed Central Ltd.

This is an Open Access article distributed under the terms of the Creative Commons Attribution License (http://creativecommons.org/licenses/by/2.0), which permits unrestricted use, distribution, and reproduction in any medium, provided the original work is properly cited.

\begin{abstract}
Background: Antibiotic resistance genes are widely used for selection of recombinant bacteria, but their use risks contributing to the spread of antibiotic resistance. In particular, the practice is inappropriate for some intrinsically resistant bacteria and in vaccine production, and costly for industrial scale production. Non-antibiotic systems are available, but require mutant host strains, defined media or expensive reagents. An unexplored concept is over-expression of a host essential gene to enable selection in the presence of a chemical inhibitor of the gene product. To test this idea in E. coli, we used the growth essential target gene fabl as the plasmid-borne marker and the biocide triclosan as the selective agent.

Results: The new cloning vector, pFab, enabled selection by triclosan at I $\mu \mathrm{M}$. Interestingly, pFab out-performed the parent $\mathrm{PUCl}$ 9-ampicillin system in cell growth, plasmid stability and plasmid yield. Also, pFab was toxic to host cells in a way that was reversed by triclosan. Therefore, pFab and triclosan are toxic when used alone but in combination they enhance growth and plasmid production through a gene-inhibitor interaction.
\end{abstract}

Conclusion: The fabl-triclosan model system provides an alternative plasmid selection method based on essential gene over-expression, without the use of antibiotic-resistance genes and conventional antibiotics.

\section{Background}

Antibiotic resistance marker genes are commonly used to select and maintain recombinant bacteria in the presence of antibiotics. However, the use of antibiotics is undesirable for manufacturing gene therapy products $[1,2]$. Also, the introduction of antibiotic resistance genes into biohazardous strains is not recommended [3], and antibiotic selection fails in bacteria that are naturally resistant $[3,4]$. Finally, the use of antibiotics can be costly in industrial scale production, especially in the case of enzymatic depletion of antibiotic during culture. An alternative sys- tem should avoid antibiotic resistance markers and therapeutic antibiotics, be cost effective, but still be convenient, robust and flexible.

A variety of strategies for antibiotic-free selection have been developed, but none are widely used in bacteria. The first reported non-antibiotic system involves an auxotrophic bacterial strain and complementation using a plasmid-encoded biosynthesis gene, such that only transformants grow on defined media lacking the nutrient [5]. Another system is termed repressor-titration, where the lac 
operator functions as the vector-borne selection marker, which de-represses a modified chromosomal essential gene [6]. A third general approach is to alter the expression of growth essential genes using synthetic [7] or expressed antisense sequences [8]. Unfortunately, these and other existing non-antibiotic systems suffer from a need for mutant host strains or expensive reagents, and in some cases low efficiency.

It has been observed that over-expression of a growth essential gene results in reduced susceptibility to the gene product inhibitor [9]. Here we considered a strategy based on an essential gene as the plasmid-borne marker and a specific protein level inhibitor as the selective agent. To test the idea, we chose fabI-triclosan as a model combination because it is a well-studied gene-inhibitor pair: FabI (enoyl ACP reductase) catalyzes fatty acid elongation [10] and confers reduced susceptibility to triclosan when overexpressed in E. coli [11,12]; triclosan inhibits FabI through binding at the ACP-enoyl substrate site, forming a stable FabI/NAD+/triclosan ternary complex [13]. In addition, triclosan is a biocide that fulfils the criteria of a non-antibiotic [14]. It is stable, easy to handle, inexpensive and approved for use in many hygiene, household and industrial applications $[15,16]$.

Although triclosan is not used as a systemic therapeutic, it is important to consider possible risks associated with new applications. Most importantly, there is concern that triclosan use may contribute to antibiotic resistance [17]; indeed, resistant mutants can be generated in the laboratory [18-20]. However, studies of bacteria in non-laboratory conditions exposed to biocide-concentrations of triclosan did not find a correlation between antibiotic resistance and decreased triclosan susceptibility [21-23]. Regulatory agencies continue to approve the use of triclosan in domestic and clinical products $[24,25]$, and it appears to be a comparatively safe choice as a selection agent.

In this study, we tested fabI-marker plasmid selection by triclosan and observed efficient and stable selection. The new system out-performed the parent antibiotic system in growth and plasmid production in the presence of triclosan. Cells containing the fabI-marker plasmid displayed toxic effects in the absence of triclosan, suggesting an "addictive" effect, which may aid plasmid containment.

\section{Methods}

\section{Bacterial strains, plasmids and media}

The E. coli strains used in this study were DH5a (Invitrogen), XL1-Blue (Strategene), HB101, BL21 (Strategene) and K12 (Coli Genetics Stock Center, Yale U.). Plasmids were pUC19 (New England Biolabs), pBAD18 and
pBAD18s (National Institute of Genetics, Japan). Media were SOC and LB (GIBCOBRL) supplemented with ampicillin (LBA, $100 \mu \mathrm{g} / \mathrm{ml}$ ampicillin, (Sigma)), triclosan (LBT, $1 \mu \mathrm{M}$ triclosan; Ciba) and arabinose (Sigma). Triclosan was used as a $1 \mathrm{M}$ stock in DMSO.

\section{Construction of pFab, pUCFA and pBFab}

An EagI site was created at nucleotide position 1621 of pUC19 (New England Biolabs), immediately downstream of bla, by PCR with primers ( 5 'cgtcggccgttaccaatgcttaatcag and 5 'cgecggccggaccaagtttactcatat). The amplicon was digested with EagI (New England Biolabs), ligated with T4 DNA ligase and transformed into DH5 $\alpha$ for propagation. To construct pFab, the bla gene was excised from pUC19 with SspI and EagI, and replaced with fabI together with its promoter, which was amplified from $\mathrm{K} 12$ genomic DNA using the primers 5 'ccggatatcgtgctggagaatattcg and 5 'gcgcggccgttatttcagttcgagttcgtt. The amplicon was digested with EcoRV and EagI and used to create the pFab vector. E. coli DH5a were transformed with $\mathrm{pFab}$ and plated onto LB with $0.5-5 \mu \mathrm{M}$ triclosan. Transformants were subsequently maintained in $1 \mu \mathrm{M}$ triclosan (LBT). The fabI gene was also cloned into pUC19 at SphI and BamHI within the MCS (pUCFA) in a similar way using the primers 5 'ccggcatgcgtgctggagaatattcg and 5 'ccggatccgattatttcagttcgagt. Competent E. coli DH5 $\alpha$ were transformed with pUCFA and plated onto LBA and LBT.

To induce expression of $f a b I$ from $\mathrm{P}_{\mathrm{BAD}}$, the $f a b I$ amplicon generated from primers $(5$ 'cggaattcgaatgggttttctttccgg and 5 'cctctagagattatttcagttcgagt) was digested with EcoRI and $\mathrm{Xba \textrm {I }}$ (New England Biolabs) and cloned into pBAD18s, which was similarly digested, to yield pBFab1. Expression of $f a b I$ in pBAD18 required a Shine Dalgarno sequence, which was predicted to be uaagga at position -13 relative to the start codon. Primers ( 5 'cggaattctcaacaataaggattaaagc and $5^{\prime}$ cctctagagattatttcagttcgagt) were used for amplification of $f a b I$ with its Shine Dalgarno sequence, and cloned into pBAD18 to yield pBFab6. E. coli DH5a were transformed with the pBFab plasmids and plated onto LBA, LBT and LBT with $0.2 \%(\mathrm{w} / \mathrm{v})$ arabinose.

\section{Plasmid and transformant properties}

Transformation efficiencies of chemically competent DH5 $\alpha$ cells were determined as recommended by the manufacturer (Invitrogen).

Plasmid yields were determined from five clones of pUC19 and pFab. Plasmids were isolated from one $\mathrm{ml}$ overnight cultures grown under selection using a miniprep kit (Qiagen) and quantified by $\mathrm{OD}_{260}$ readings. Plasmids (50 ng) were digested with BamHI and electrophoresed in a $1 \%$ agarose gel. 
Plasmid stability was assayed either in the presence or absence of selection. Overnight cultures grown under selection at $37^{\circ} \mathrm{C}$ with shaking were diluted $1000 \times$ in 5 $\mathrm{ml} \mathrm{LB}$ with or without selection, and aliquots of the time zero cultures were diluted and plated onto LB plates containing X-Gal $(20 \mu \mathrm{g} / \mathrm{ml}$, Saveen $)$. The time zero cultures were grown and diluted as above at 24 and $48 \mathrm{~h}$, and aliquots of the $48 \mathrm{~h}$ cultures were plated. The ratios of blue colonies to total colonies on LB plates with X-Gal $(20 \mu \mathrm{g} /$ $\mathrm{ml}$ ) were determined at $0 \mathrm{~h}$ and $48 \mathrm{~h}$, from which \% plasmid-bearing cells at $48 \mathrm{~h}$ were calculated. Five independent clones of pUC19 and pFab were used to provide replicates.

Plasmid abundance was determined in two ways. First, to compare band intensities of genomic (gDNA) to plasmid DNA (pDNA) in an agarose gel, total genomic DNA was extracted from five different clones of pUC19 and pFab clones grown under selection for $16 \mathrm{~h}$. Five cultures of $\mathrm{K} 12$, derived from five single colonies, were grown in LB for DNA extraction using the Bacterial GenElute system (Sigma) for one ml of overnight culture. Total DNA (10 $\mu \mathrm{l})$ was electrophoresed in a $1 \%$ agarose gel, stained with ethidium bromide and scanned using a Typhoon 9400 (Amersham Biosciences). Band intensities were determined by using ImageQuant software (Amersham Biosciences). Second, relative quantitative PCR (qPCR) was carried out by using the plasmid lacZ $\alpha$ gene as the target gene and single copy chromosomal $d x$ s as the reference gene [26]. K12 gDNA containing a single copy of lacZ $\alpha$ and $d x s$ was used as a calibrator. Primers amplifying the target gene (5'gtgctgcaaggcgattaagtt and 5' cactggccgtcgttttacaa), and reference gene ( 5 'cgagaaactggcgatcctta and 5 'cttcatcaagcggtttcaca) were validated for similar amplification efficiencies. Real time data analyses were carried out by the $2^{-\Delta \Delta C T}$ method for relative qPCR [27]. Total DNA concentrations were determined by $\mathrm{OD}_{260}$ absorbance for qPCR. Each $25 \mu \mathrm{l}$ of PCR reaction contained 12.5 $\mu \mathrm{l}$ of SYBR Green PCR buffer (Eurogentec), $100 \mathrm{nM}$ of each primer (Biomers) and $5 \mathrm{ng}$ total DNA.

Growth rates were calculated from the exponential phase of growth [28], which was monitored as increased $\mathrm{OD}_{550}$ over time by the VERSAmax spectrophotometer (Molecular Devices). An overnight culture (16 h), standardized by $\mathrm{OD}_{550}$ to yield approximately $7 \times 10^{5} \mathrm{cfu} / \mathrm{ml}$, was grown in $200 \mu$ l volumes per well in a 96-well plate for $24 \mathrm{~h}$ with agitation for $5 \mathrm{~s}$ every $5 \mathrm{~min}$, when $\mathrm{OD}_{550}$ readings were taken. Triclosan was added to give $0-2 \mu \mathrm{M}$ Triclosan and $1 \%$ DMSO final concentration.

The host range of pFab within commonly used E. coli cloning strains was tested by transformation of XL1-Blue (Stratagene), HB101 and BL21, followed by selection on LBT plates. Plasmid DNA integrity and abundance was determined by plasmid extraction, digestion of $100 \mathrm{ng}$ DNA with BamHI and fractionation in a $1 \%$ agarose gel.

\section{Inducible expression of fabl}

Clones of pBFab1 and pBFab6 were grown in LBA for 16 $\mathrm{h}$, diluted to approximately $5-9 \times 10^{6} \mathrm{cfu} / \mathrm{ml}$ in LBT and aliquots of $180 \mu \mathrm{l}$ were added to wells in a 96 well plate. Arabinose was added to a final concentration of $0-5 \%$ and the final volume per well was made up to $200 \mu \mathrm{l}$ with water. Clones of pBAD18 and pBAD18s were included as controls. Cultures were grown for $24 \mathrm{~h}$ in the VERSAmax spectrophotometer (Molecular Devices) with agitation for $5 \mathrm{~s}$ every $5 \mathrm{~min}$, followed by an $\mathrm{OD}_{550}$ reading. The growth rate at each arabinose concentration was calculated as described above.

\section{Cell viability}

DH $5 \alpha /$ pFab were grown overnight in the absence or presence of $0.5-2 \mu \mathrm{M}$ triclosan and subjected to SYTOX green staining and flow cytometry, as previously described [29]. DH5 $\alpha /$ pUC19 was included as a control to determine staining levels of live and heat-treated dead cells. Samples were excited with a $488 \mathrm{~nm}$ air-cooled argon ion laser in the CyFlow SL flow cytometer (Partec GmbH). Threshold settings were enabled on forward scatter to exclude cell debris. The forward and side scatter dot plot was used to identify and gate cell populations. Fluorescence was measured at $520 \mathrm{~nm}$. Viable and dead cell populations were counted using the Partec, FloMax software version 2.4e.

\section{Growth competition}

Growth competition between DH5 $\alpha$ and plasmid bearing cells was carried out as previously described, with modifications [30]. Overnight ( $24 \mathrm{~h}$ ) cultures of DH5 $\alpha$, DH5 $\alpha /$ pUC19 and DH5 $\alpha /$ pFab were prepared in LB, LBA and LBT, respectively. Equal volumes of DH5 $\alpha$ and plasmid bearing cultures were mixed and diluted 1:100 in $10 \mathrm{ml}$ fresh LB for further growth. An aliquot of the diluted mixed culture was simultaneously plated onto selective and non-selective media for cell counts. After the mixed culture was incubated for $24 \mathrm{~h}$ with shaking at $37^{\circ} \mathrm{C}$, it was diluted 1:100 in $10 \mathrm{ml}$ of fresh LB for further growth and plated, as above. This procedure was repeated 5 times over six days. The numbers of ampicillin or triclosan resistant colonies were scored relative to the total CFUs.

\section{Results}

\section{Vectors containing fabl enable triclosan selection}

To test the potential of $f a b I$ as a selective marker for cloning, two vectors derived from pUC19 were constructed. First, we constructed pFab, where fabI together with its native promoter replaces the ampicillin resistance gene (bla) in pUC19. Second, to enable selection with triclosan or ampicillin, we constructed pUCFA, which contains the fabI cassette cloned into the pUC19 MCS (Figure 1A). E. 
A
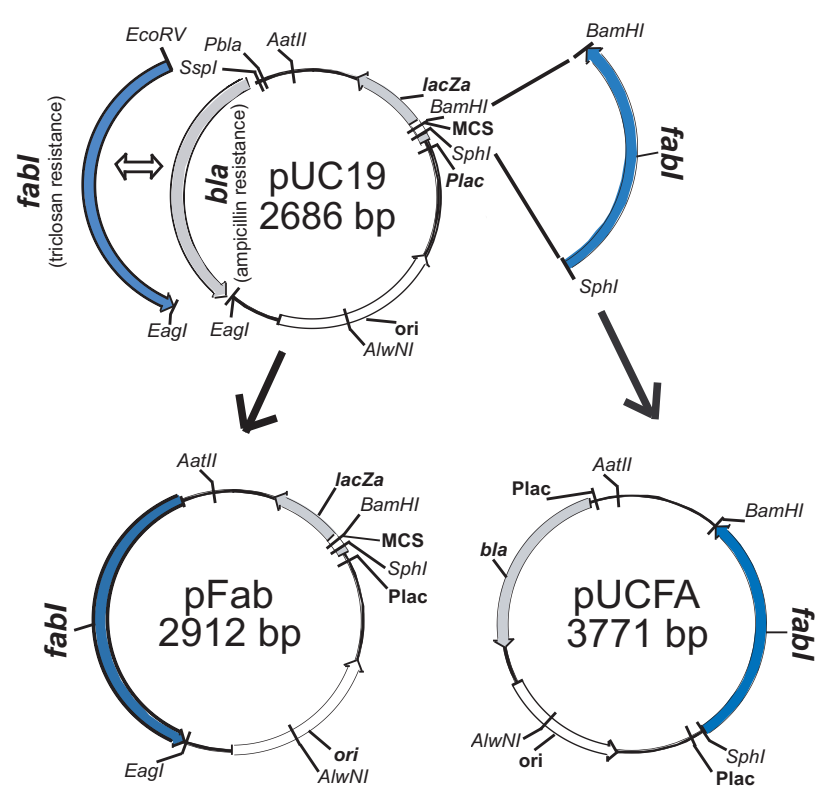

B

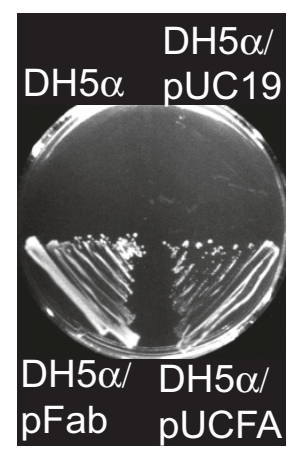

Triclosan (LBT)

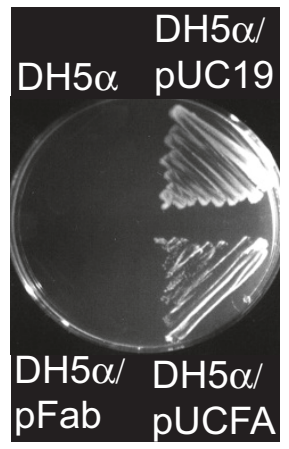

Ampicillin (LBA)

C

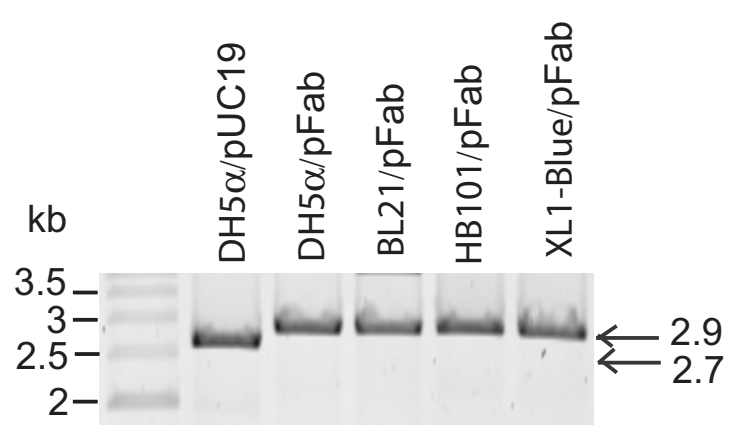

Figure I

Figure I

Vector construction and triclosan selection. (A) The bla gene in pUCI9, which confers ampicillin resistance, was replaced with fabl and its promoter region (pFab). The pUCI 9 multiple cloning site (MCS) is retained, however Hincll, HindlII and Pstl are not unique in pFab. The fabl cassette in pFab can be transferred to other pUC-derived plasmids using the Aatll and AlwNI restriction sites. The fabl cassette was also inserted into the MCS of PUCI 9 to obtain PUCFA. All plasmids are available from the authors. (B) Growth of pFab and PUCFA clones on LBT and LBA plates. (C) Plasmids propagated in different $E$. coli hosts were digested with BamHI and analyzed by gel electrophoresis.

coli strain DH5 $\alpha$ was transformed with pFab, pUCFA or pUC19 and transformants were selected on LB plates containing triclosan (LBT) or ampicillin (LBA). As anticipated, fabI inserts enabled selection on triclosan containing plates. Colonies formed on LBT were more variable in size than colonies on LBA. However, triclosanresistant colonies of all sizes maintained resistance (Figure 1B) and displayed uniform colony morphologies upon replating. In contrast to pUC19, we did not observe plasmid-free colonies or satellite colonies when using pFab and pUCFA. Therefore, the fabI-triclosan system enables non-antibiotic selection and maintains stable recombinant strains.

To test whether fabI-triclosan selection could function well in other E. coli strains, pFab was transformed into strains BL21, HB101 and XL1-blue. Similar to the results in DH5 $\alpha$, we observed efficient selection and high yield plasmid production (Figure 1C). To test whether fabI can enable triclosan selection in other vectors, the pFab cassette was inserted into the multiple cloning site of pGEM$3 \mathrm{Zf}$ and also used to replace the ampicillin resistance gene in the low copy number vector pBR322. In both cases, triclosan resistant colonies were selected (data not shown). Therefore, the fabI-triclosan system enables efficient selection in commonly used vectors and E. coli strains.

To confirm that $f a b I$ expression was the main mechanism mediating triclosan resistance and not point mutations within chromosomal fabI [12], expression of fabI was placed under the control of the $\mathrm{P}_{\mathrm{BAD}}$ promoter in pBAD18s (pBFab1) and pBAD18 (pBFab6) [31]. The pBFab1 and pBFab6 strains were tested for resistance following fabI induction. In the absence of the inducer arabinose, no growth was observed in LBT broth, but growth rates increased with increasing arabinose concentrations up to $0.4 \%$ (Figure 2). Therefore, pFab-mediated resistance to triclosan is due to expression of $f a b I$. 


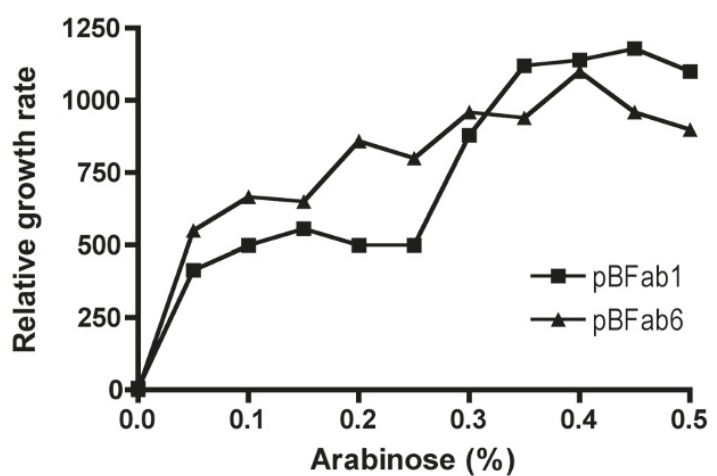

Figure 2

Effect of fabl induction on triclosan resistance. Triclosan resistance mediated by arabinose-induced overexpression of fabl. Exponential growth rates are for $\mathrm{DH} 5 \alpha /$ PBFabI and DH5 $\alpha /$ pBFab6 relative to DH5 $\alpha / \mathrm{pBAD} / 8$ s and DH5 $\alpha / p B A D \mid 8$, respectively.

\section{Characterization of pFab}

After selection of pFab transformants with triclosan, we characterized the general properties of $\mathrm{pFab}$ as a cloning vector, with pUC19 included for comparison (Table 1). Plasmid preparation yield of pFab was $43 \%$ greater than pUC19 (Table 1) in E. coli DH5 $\alpha$ transformants. Also, the copy number of pFab was $38 \%$ and $40 \%$ greater than that of pUC19, as measured by qPCR and plasmid to genomic DNA abundance (Table 1; Figure 3A \& B), respectively. Therefore, pFab displayed higher yield and copy number relative to the parent vector.

To assess plasmid stability, we first scored the number of triclosan resistant colony forming units relative to total colony forming units. Surprisingly, we observed more colonies on LBT than on LB plates. This indicated high plasmid stability in the presence of triclosan, but some form of pFab-mediated toxicity in the absence of triclosan. Indeed, measurements of plasmid stability using an alternative $\alpha$-complementation assay revealed that $\mathrm{pFab}$ was more stable than pUC19 under selection (Table 1). Therefore, while pFab over-expression clearly conferred triclosan resistance, it also appeared to confer a requirement for the biocide.

\section{Effects of pFab and triclosan on E. coli growth, survival and fitness}

To further investigate the interaction between $f a b I$ and triclosan, we first examined culture growth profiles. While ampicillin had little effect on DH5 $\alpha$ /pUC19 cultures, the final optical density of $\mathrm{DH} 5 \alpha / \mathrm{pFab}$ was highest in the presence of triclosan and the exponential phase growth rates of $\mathrm{DH} 5 \alpha / \mathrm{pFab}$ cultures were faster than $\mathrm{DH} 5 \alpha /$ pUC19 cultures under selection, whereas the inverse was
A
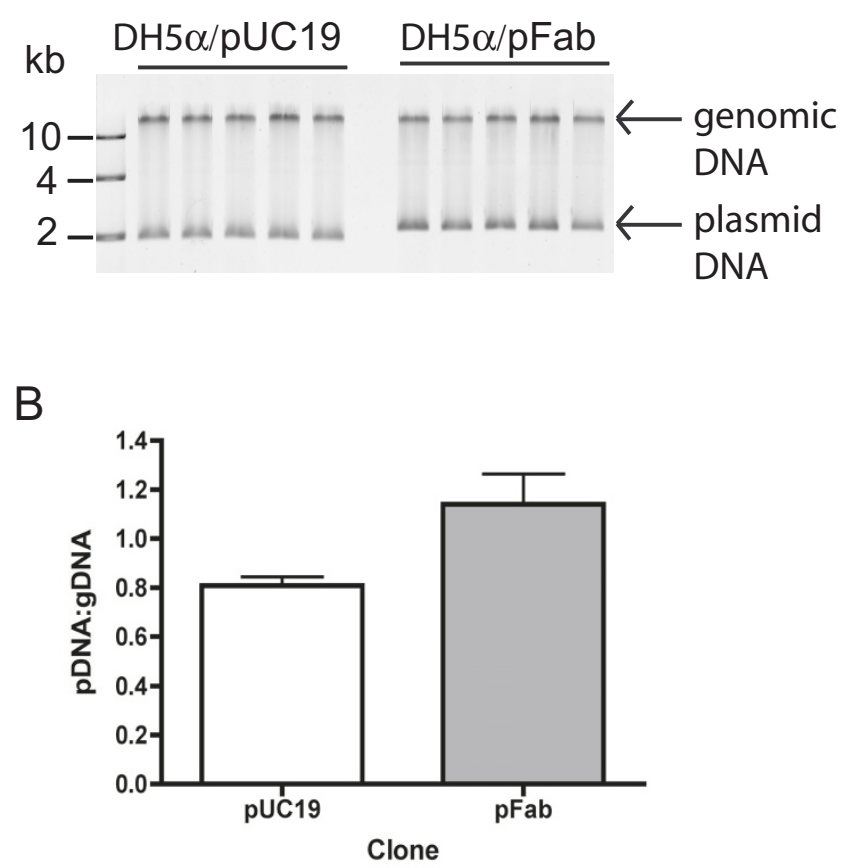

Figure 3

Plasmid production of pFab transformants. (A) Agarose gel electrophoresis of total DNA isolated from five clones of pUCI 9 and pFab. Bands were quantified by using ImageQuant software to calculate ratio of PDNA to gDNA as a measure of copy number in pUCI 9 and pFab. (B) Mean ratios of pDNA:gDNA of pUCI 9 and pFab from $(\mathbf{A})$.

observed without selection (Figure 4A; Table 1). We next determined $\mathrm{DH} 5 \alpha / \mathrm{pFab}$ culture growth rates in the presence of a range of triclosan concentrations $(0-2 \mu \mathrm{M})$. As pFab was unstable without triclosan, DH5 $\alpha /$ pUCFA grown in LBA was included to prevent the growth of competing plasmid-free cells. Growth rates of pUCFA and pFab clones were lowest without triclosan and increased with triclosan addition up to $1 \mu \mathrm{M}$, indicating a triclosandependent fitness rescue in cells that carry pFab (Figure 4B). Similarly, we observed that the proportion of dead cells decreased with increasing triclosan concentrations. Fluorescence microscopy of the SYTOX stained cells [29] revealed elongated and dead cells in the absence of triclosan, and elongated and viable cells in $1 \mu \mathrm{M}$ triclosan (data not shown). The ratio of live to dead cells indicated by SYTOX staining was then quantified by flow cytometry [29]. The results again showed that triclosan reduced the number of dead cells, indicating that pFab transformants were rescued by triclosan (Figure $4 \mathrm{C}$ ). Therefore, pFab is toxic but this toxicity is suppressed by triclosan. 
Table I: Properties of pFab and pUCI 9

\begin{tabular}{|c|c|c|}
\hline Parameters $^{\mathrm{a}}$ & $\mathrm{pUCl9}$ & pFab \\
\hline Transformation efficiency $(\mathrm{CFU} / \mu \mathrm{g})$ & $5.5 \times 10^{7} \pm 1.2 \times 10^{7}$ & $2.2 \times 10^{7} \pm 3.6 \times 10^{6}$ \\
\hline Plasmid yieldc $(\mu \mathrm{g} / \mathrm{ml})$ & $30 \pm 2.1$ & $42.9 \pm 3.5$ \\
\hline Copy numberd & $|4| \pm 25$ & $200 \pm 33$ \\
\hline \multicolumn{3}{|l|}{ Stability (\%) } \\
\hline With selectione & $85.3 \pm 10.8$ & $99.5 \pm 1.5$ \\
\hline Without selection ${ }^{f}$ & $56.7 \pm 17.7$ & $54.5 \pm 14.9$ \\
\hline \multicolumn{3}{|l|}{ Relative growth rateg $(\Delta O D / \Delta t)$} \\
\hline With selection & 1 & $1.2 \pm 0.07$ \\
\hline Without selection & $\mathrm{I} . \mathrm{I} \pm 0.05$ & $0.6 \pm 0.02$ \\
\hline
\end{tabular}

a Determined from five replicate cultures.

b In chemically competent $\mathrm{DH} 5 \alpha$ cells.

c Plasmid yield from I ml of $18 \mathrm{~h}$ culture, determined by $\mathrm{OD}_{260}$ absorbance.

d Determined from the copy ratio of lac $Z \alpha$ to $d x s$ by qPCR.

e Percentage of plasmid-bearing cells at $48 \mathrm{~h}$ in cultures grown with selection.

fPercentage of plasmid-bearing cells at $48 \mathrm{~h}$ in cultures grown without selection.

8 Change in $\mathrm{OD}_{550}$ over time of $\mathrm{DH} 5 \alpha / \mathrm{pUC} 19$ cultures in LB and DH5 $\alpha / \mathrm{pFab}$ in LB or LBT, relative to control DH5 $\alpha / \mathrm{pUCI} 9$ cultures in LBA.

To test whether triclosan resistance is likely to persist outside intended applications, we assessed the fitness of DH5/pUC19 and DH5/pFab strains in mixed culture with the plasmid-free parent strain. A competitive fitness assay, conducted in the absence of selection, showed that pFab persistence was weaker than that of pUC19 during co-culture over six days (Figure 4D). In other words, the rate of pFab loss was faster than pUC19 loss and therefore, in the absence of selection, pFab is less stable and less competitive than pUC19.

\section{Discussion}

We describe over-expression of a growth essential gene conferring resistance to a specific protein inhibitor as a plasmid selection system in E. coli, using fabI and triclosan as an example. As well as avoiding the use of antibiotic resistance genes and antibiotics, pFab transformants showed improved growth, yield and gene containment. These improvements appear to be due to the mechanism of inhibition [13] and a balance of toxic gene-inhibitor levels required for cell survival [32] and selection [33]. Similar interactions in other systems suggest that geneinhibitor reciprocal suppression may be a common mechanism. For example, reciprocal or mutual suppression has been described between two genes [34] and chemical inhibitors [35]. Also, antibiotic-dependent strains have been described for bacteria isolated from laboratories $[36,37]$ and clinics [38].

The new selection system relies on an endogenous E. coli gene and a widely used biocide. Nevertheless, the relative safety of triclosan and fabI in this application should be considered. Triclosan is approved by regulatory authorities in the EU and the USA for many applications [24,25] and an association between bacterial triclosan resistance and antibiotic susceptibility, though suggested, has not been found in practice $[21-23,39,40]$. In the laboratory, spontaneous triclosan resistance in $E$. coli resulting from exposure to low triclosan concentration has been observed, where three point mutations in fabI increased MIC by up to 95 fold [12]. Such mutations may arise during triclosan selection. However, in our experiments, triclosan resistance was dependent on expression of plasmid-borne fabI (Figure 2), and blue-white selection of recombinant $E$. coli indicated mostly pFab-carrying cells (blue) and very few spontaneous resistant mutants (Table 1). In the environment, triclosan resistance has been slow to emerge compared to antibiotic resistance [21,23], possibly due to poor solubility of triclosan [41], rapid degradation of triclosan $[22,42,43]$, low competitive fitness of FabI mutants [12], and the tripartite nature of the FabI/ $\mathrm{NADPH} /$ triclosan complex. Furthermore, the spread of pFab outside of intended applications should be limited due to low plasmid stability, poor competitive fitness and cell toxicity in the absence of triclosan. Indeed, FabI is stringently regulated within the fatty acid biosynthesis pathway [32], and de-regulation inhibits cell growth and viability $[44,45]$. Nevertheless, it is possible that pFab could transfer horizontally and thus induce resistance to triclosan in wild-type bacteria, and standard precautions in the handling of genetically modified microorganisms should be maintained.

In large scale production of proteins, plasmid stability without selection is a pre-requisite because residual antibiotics are undesirable. In this regard, the finding that pFab requires triclosan for plasmid stability could be a disadvantage. However, the amount of triclosan required is much less compared to that in antibiotic selection (typically $2 \%$ ), and a level of residual triclosan may be permissible, given its approval for use in non-prescription medicines and hygiene products. Therefore, the need for 

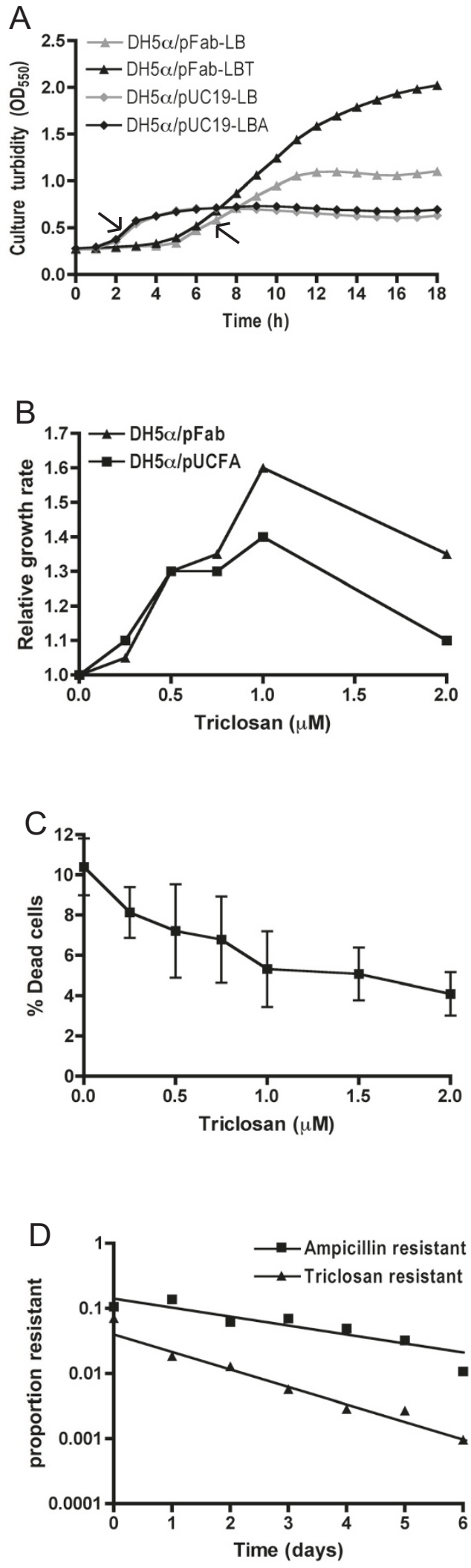

Figure 4
Figure 4

Effect of triclosan on growth, viability and fitness pFab transformants. (A) Growth curves for $\mathrm{DH} 5 \alpha$ / pUCI 9 and $\mathrm{DH} 5 \alpha / \mathrm{pFab}$ cultures grown with and without selection. Growth rates were determined from the exponential phase, indicated by arrows. (B) Reduced fitness with fabl over-expression and its suppression by triclosan. Growth rates of pFab and pUCFA clones in LB and LBA, respectively, were calculated relative to DH5 $\alpha / p U C 19$ in LBA. (C) Flow cytometric determination of dead cell numbers, as a measure of cell toxicity, in $\mathrm{DH} 5 \alpha / \mathrm{pFab}$ cultures grown in the presence of a range of triclosan concentrations. (D) Growth competition between $\mathrm{DH} 5 \alpha$ and $\mathrm{DH} 5 \alpha / \mathrm{pUC} 19$ or $\mathrm{DH} 5 \alpha / \mathrm{pFab}$. The $\log 10$ ratio of plasmid-bearing cells to total number of cells against time represents the rate of plasmid loss in mixed cell populations. The data is representative of two independent experiments.

stability in the absence of selection may prove less important with this system. Nevertheless, pFab stability is a potential problem and vector construct or process adjustments may be needed during scale-up.

\section{Conclusion}

In summary, this study provides an example of how essential genes can be used in combination with non-antibiotic inhibitors to select and maintain recombinant bacteria. It may be possible to apply triclosan selection to other bacteria such as Staphylococcus aureus [46] and Mycobacterium smegmatis [47], which have homologues of FabI. Other inhibitors of FabI have been described [48,49] and, depending on bacterial resistance development, certain triclosan analogues may be preferred for use in this plasmid selection system. On the other hand, bacteria that have divergent enoyl-ACP reductase, such as Bacillus subtilis (FabL) [50] and Streptococcus pneumoniae (FabK) [51], and species that are intrinsically resistant to triclosan, such as Pseudomonas aeruginosa [18], are not suitable for fabI-triclosan plasmid selection. For such species, it would be interesting to test additional essential gene inhibitor combinations to expand the plasmid selection strategy described here. The pFab and triclosan system is potentially attractive for production of recombinant proteins, because it can be used to increase plasmid copy number and yield. In addition, pFab and its derivatives may be suitable for manufacturing biopharmaceuticals and gene therapy products, and in other applications that require an absence of antibiotic resistance sequences or antibiotic residues.

\section{Authors' contributions}

SG carried out all experimental work and data analyses except for flow cytometry, participated in design of study and drafted the manuscript. LG conceived the study, car- 
ried out flow cytometry and FACS analysis, and helped with design and drafting the manuscript. All authors have read and approved the final manuscript.

\section{Acknowledgements}

We thank the Swedish Institute for a Guest Scholarship for SG and the Swedish Research Council and University of London Central Research Fund for support. We also thank Thomas Bentin, Omid Faridani, Abbas Nikravesh, Mónica Marta, Joshua Fink and Rikard Dryselius for help and comments.

\section{References}

I. Points to consider in the production and testing of new drugs and biologicals produced by recombinant DNA technology [http://www.fda.gov/cber/gdlns/ptcdna.htm]

2. Validation of growth-based rapid microbiological methods for sterility testing of cellular and gene therapy products [http://www.fda.gov/cber/gdlns/stercgtp.htm]

3. Titball RW, Sjostedt A, Pavelka MS Jr, Nano FE: Biosafety and selectable markers. Ann N Y Acad Sci 2007, I I 05:405-4I7.

4. Fajardo A, Martinez-Martin N, Mercadillo M, Galan JC, Ghysels B, Matthijs S, Cornelis P, Wiehlmann L, Tummler B, Baquero F, Martinez $\mathrm{L}$ : The neglected intrinsic resistome of bacterial pathogens. PLOS ONE 2008, 3:e1619.

5. Gonzalez A, Davila G, Calva E: Cloning of a DNA sequence that complements glutamine auxotrophy in Saccharomyces cerevisiae. Gene 1985, 36:123-129.

6. Cranenburgh RM, Hanak JA, Williams SG, Sherratt DJ: Escherichio coli strains that allow antibiotic-free plasmid selection and maintenance by repressor titration. Nucleic Acids Res 200I, 29:E26.

7. Dryselius R, Nekhotiaeva N, Nielsen PE, Good L: Antibiotic-free bacterial strain selection using antisense peptide nucleic acid. Biotechniques 2003, 35:1060-1064.

8. Mairhofer J, Pfaffenzeller I, Merz D, Grabherr R: A novel antibiotic free plasmid selection system: advances in safe and efficient DNA therapy. Biotechnol / 2008, 3:83-89.

9. $\mathrm{Xu} \mathrm{HH}$, Real L, Bailey MW: An array of Escherichia coli clones over-expressing essential proteins: a new strategy of identifying cellular targets of potent antibacterial compounds. Biochem Biophys Res Commun 2006, 349:1250-1257.

10. Bergler H, Wallner P, Ebeling A, Leitinger B, Fuchsbichler S, Aschauer H, Kollenz G, Hogenauer G, Turnowsky F: Protein EnvM is the NADH-dependent enoyl-ACP reductase (Fabl) of Escherichia coli. I Biol Chem 1994, 269:5493-5496.

II. Heath RJ, Yu YT, Shapiro MA, Olson E, Rock CO: Broad spectrum antimicrobial biocides target the Fabl component of fatty acid synthesis. J Biol Chem 1998, 273:30316-30320.

12. McMurry LM, Oethinger M, Levy SB: Triclosan targets lipid synthesis. Nature 1998, 394:53।-532.

13. Heath RJ, Rubin JR, Holland DR, Zhang E, Snow ME, Rock CO: Mechanism of triclosan inhibition of bacterial fatty acid synthesis. J Biol Chem 1999, 274: I I I 10-IIII4.

14. Russell AD: Biocide use and antibiotic resistance: the relevance of laboratory findings to clinical and environmental situations. Lancet Infect Dis 2003, 3:794-803.

15. Panagakos FS, Volpe AR, Petrone ME, DeVizio W, Davies RM, Proskin HM: Advanced oral antibacterial/anti-inflammatory technology: A comprehensive review of the clinical benefits of a triclosan/copolymer/fluoride dentifrice. J Clin Dent 2005, 16(Suppl):SI-19.

16. Wohlrab J, Jost G, Abeck D: Antiseptic efficacy of a low-dosed topical triclosan/chlorhexidine combination therapy in atopic dermatitis. Skin Pharmacol Physiol 2007, 20:71-76.

17. Aiello $A E$, Larson $E$ : Antibacterial cleaning and hygiene products as an emerging risk factor for antibiotic resistance in the community. Lancet Infect Dis 2003, 3:50I-506.

18. Chuanchuen R, Beinlich K, Hoang TT, Becher A, Karkhoff-Schweizer RR, Schweizer HP: Cross-resistance between triclosan and antibiotics in Pseudomonas aeruginosa is mediated by multidrug efflux pumps: exposure of a susceptible mutant strain to triclosan selects nfxB mutants overexpressing MexCD-OprJ. Antimicrob Agents Chemother 200I, 45:428-432.
19. Braoudaki M, Hilton AC: Adaptive resistance to biocides in Salmonella enterica and Escherichia coli 0157 and cross-resistance to antimicrobial agents. J Clin Microbiol 2004, 42:73-78.

20. Karatzas KA, Webber MA, Jorgensen F, Woodward MJ, Piddock LJ, Humphrey TJ: Prolonged treatment of Salmonella enterica serovar Typhimurium with commercial disinfectants selects for multiple antibiotic resistance, increased efflux and reduced invasiveness. J Antimicrob Chemother 2007, 60:947-955.

21. Cole EC, Addison RM, Rubino JR, Leese KE, Dulaney PD, Newell MS, Wilkins J, Gaber DJ, Wineinger T, Criger DA: Investigation of antibiotic and antibacterial agent cross-resistance in target bacteria from homes of antibacterial product users and nonusers. J Appl Microbiol 2003, 95:664-676.

22. McBain AJ, Bartolo RG, Catrenich CE, Charbonneau D, Ledder RG, Price BB, Gilbert P: Exposure of sink drain microcosms to triclosan: population dynamics and antimicrobial susceptibility. Appl Environ Microbiol 2003, 69:5433-5442.

23. Beier RC, Duke SE, Ziprin RL, Harvey RB, Hume ME, Poole TL, Scott HM, Highfield LD, Alali WQ, Andrews K, et al:: Antibiotic and Disinfectant Susceptibility Profiles of Vancomycin-Resistant Enterococcus faecium (VRE) Isolated from Community Wastewater in Texas. Bull Environ Contam Toxicol 2008.

24. Opinion on triclosan resistance [http://www.europa.eu.int/ comm/food/fs/sc/ssc/out269 en.pdf]

25. Drug approvals [http://www.fda.gov/cder/da/da $201 . h t m]$

26. Lee C, Kim J, Shin SG, Hwang S: Absolute and relative QPCR quantification of plasmid copy number in Escherichia coli. J Biotechnol 2006, I 23:273-280.

27. Livak KJ, Schmittgen TD: Analysis of relative gene expression data using real-time quantitative PCR and the 2(-Delta Delta C(T)) Method. Methods 200I, 25:402-408.

28. Nilsson Al, Zorzet A, Kanth A, Dahlstrom S, Berg OG, Andersson DI: Reducing the fitness cost of antibiotic resistance by amplification of initiator tRNA genes. Proc Natl Acad Sci USA 2006 | 03:6976-698|

29. Roth BL, Poot M, Yue ST, Millard PJ: Bacterial viability and antibiotic susceptibility testing with SYTOX green nucleic acid stain. Appl Environ Microbiol I997, 63:242 I-2431.

30. Lenski RE, Simpson SC, Nguyen TT: Genetic analysis of a plasmid-encoded, host genotype-specific enhancement of bacterial fitness. J Bacteriol 1994, I 76:3 | 40-3|47.

31. Guzman LM, Belin D, Carson MJ, Beckwith J: Tight regulation, modulation, and high-level expression by vectors containing the arabinose PBAD promoter. J Bacteriol 1995, 177:4| 2 I-4I30.

32. Heath RJ, Rock CO: Regulation of fatty acid elongation and initiation by acyl-acyl carrier protein in Escherichia coli. J Biol Chem 1996, 271:1833-1836.

33. Ward WH, Holdgate GA, Rowsell S, McLean EG, Pauptit RA, Clayton E, Nichols WW, Colls JG, Minshull CA, Jude DA, et al:: Kinetic and structural characteristics of the inhibition of enoyl (acyl carrier protein) reductase by triclosan. Biochemistry 1999, 38:125|4-12525

34. Weitao T, Nordstrom K, Dasgupta S: Mutual suppression of mukB and seqA phenotypes might arise from their opposing influences on the Escherichia coli nucleoid structure. Mo Microbiol 1999, 34:157-168.

35. Yue TF, Dayton PG, Gutman AB: Mutual Suppression of the Uricosuric Effects of Sulfinpyrazone and Salicylate: a Study in Interactions between Drugs. I Clin Invest 1963, 42:1330-I339.

36. Wild DG: Reversion from erythromycin dependence in Escherichia coli: strains altered in ribosomal sub-unit association and ribosome assembly. I Gen Microbiol 1988 134:125I-1263.

37. Goldstein F, Perutka J, Cuirolo A, Plata K, Faccone D, Morris J, Sournia A, Kitzis MD, Ly A, Archer G, Rosato AE: Identification and phenotypic characterization of a beta-lactam-dependent, methicillin-resistant Staphylococcus aureus strain. Antimicrob Agents Chemother 2007, 5 I:25 I 4-2522.

38. Van Bambeke F, Chauvel M, Reynolds PE, Fraimow HS, Courvalin P: Vancomycin-dependent Enterococcus faecalis clinical isolates and revertant mutants. Antimicrob Agents Chemother 1999, 43:4I-47.

39. Aiello AE, Marshall B, Levy SB, Della-Latta P, Larson E: Relationship between triclosan and susceptibilities of bacteria isolated from hands in the community. Antimicrob Agents Chemother 2004 48:2973-2979. 
40. Gilbert P, McBain A, Sreenivasan P: Common therapeutic approaches for the control of oral biofilms: microbiological safety and efficacy. Clin Microbiol Infect 2007, I 3(Suppl 4): 17-24.

4I. Orvos DR, Versteeg DJ, Inauen J, Capdevielle M, Rothenstein A, Cunningham V: Aquatic toxicity of triclosan. Environ Toxicol Chem 2002, 21:1338-1349.

42. Federle TW, Kaiser SK, Nuck BA: Fate and effects of triclosan in activated sludge. Environ Toxicol Chem 2002, 21:1330-1337.

43. Aranami K, Readman JW: Photolytic degradation of triclosan in freshwater and seawater. Chemosphere 2007, 66:1052-1056.

44. Keating DH, Carey MR, Cronan JE Jr: The unmodified (apo) form of Escherichia coli acyl carrier protein is a potent inhibitor of cell growth. J Biol Chem 1995, 270:22229-22235.

45. Subrahmanyam S, Cronan JE Jr: Overproduction of a functional fatty acid biosynthetic enzyme blocks fatty acid synthesis in Escherichia coli. J Bacteriol 1998, 180:4596-4602.

46. Heath RJ, Li J, Roland GE, Rock CO: Inhibition of the Staphylococcus aureus NADPH-dependent enoyl-acyl carrier protein reductase by triclosan and hexachlorophene. J Biol Chem 2000, 275:4654-4659.

47. McMurry LM, McDermott PF, Levy SB: Genetic evidence that InhA of Mycobacterium smegmatis is a target for triclosan. Antimicrob Agents Chemother 1999, 43:71 I-7I 3.

48. Heerding DA, Chan G, DeWolf WE, Fosberry AP, Janson CA, Jaworski DD, McManus E, Miller WH, Moore TD, Payne DJ, et al.: I,4-Disubstituted imidazoles are potential antibacterial agents functioning as inhibitors of enoyl acyl carrier protein reductase (Fabl). Bioorg Med Chem Lett 200I, I I:206I-2065.

49. Lu H, Tonge PJ: Inhibitors of Fabl, an Enzyme Drug Target in the Bacterial Fatty Acid Biosynthesis Pathway. Acc Chem Res 2008, 4I: I I-20.

50. Heath RJ, Rock CO: A triclosan-resistant bacterial enzyme. Nature 2000, 406: $145-146$.

5I. Marrakchi H, Dewolf WE Jr, Quinn C, West J, Polizzi BJ, So CY, Holmes DJ, Reed SL, Heath RJ, Payne DJ, et al.: Characterization of Streptococcus pneumoniae enoyl-(acyl-carrier protein) reductase (FabK). Biochem J 2003, 370: 1055-1062.

Publish with Bio Med Central and every scientist can read your work free of charge

"BioMed Central will be the most significant development for disseminating the results of biomedical research in our lifetime. "

Sir Paul Nurse, Cancer Research UK

Your research papers will be:

- available free of charge to the entire biomedical community

- peer reviewed and published immediately upon acceptance

- cited in PubMed and archived on PubMed Central

- yours - you keep the copyright
BioMedcentral 